\title{
Advanced technology in the risk stratification- based strategy: The way forward to keep going
}

\author{
Roberta Assante, MD, PhD, a Emilia Zampella, $\mathrm{MD}, \mathrm{PhD},{ }^{\mathrm{a}}$ and \\ Wanda Acampa, MD, PhD ${ }^{\mathrm{a}, \mathrm{b}}$ \\ a Department of Advanced Biomedical Sciences, University Federico II, Naples, Italy \\ b Institute of Biostructure and Bioimaging, National Council of Research, Naples, Italy
}

Received May 8, 2020; accepted May 8, 2020

doi: $10.1007 / \mathrm{s} 12350-020-02198-x$

\section{See related article, pp. 2927-2936}

Myocardial perfusion imaging (MPI) represents the most common used noninvasive diagnostic procedure in patients with suspected or known coronary artery disease (CAD). Many studies in literature have been published over the years demonstrating the high accuracy of the nuclear cardiology procedures in the diagnosis of CAD. ${ }^{1}$ Nowadays, the greatest clinical impact of such procedures is linked to their prognostic value. ${ }^{2}$ In particular, in the latest years clinical research is oriented on demonstrate effectiveness of MPI for an appropriate use in imaging guided strategy in patients with suspected or known CAD. ${ }^{3}$ In this context, risk assessment represents an important step to identify patients at high risk of disease requiring a specific diagnostic and/or therapeutic approach to prevent adverse events at short and long-term follow-up. The risk of developing cardiovascular disease is influenced by the combined effects of several factors, which can have higher predictive values when combined in predictive models. In this context, gated single-photon emission computed tomography (SPECT) MPI provides important prognostic information related to both the extent and severity of myocardial perfusion abnormalities and dysfunctional left ventricular (LV) parameters. $^{2-4}$ Moreover, it is widely demonstrated that there was a linear relationship between the extent of

\footnotetext{
Reprint requests: Wanda Acampa, MD, PhD, Department of Advanced Biomedical Sciences, University Federico II, Via Pansini 5, 80131 Naples, Italy; acampa@unina.it

J Nucl Cardiol 2021;28:2937-40.

1071-3581/\$34.00

Copyright (C) 2020 American Society of Nuclear Cardiology.
}

such abnormalities and cardiac event rate. ${ }^{5}$ Moreover, a normal MPI was associated with a very low risk of cardiac events, less than $1 \%$ in the rate of adverse events, with 5 to 6 -fold increase in the presence of abnormal SPECT MPI. ${ }^{6}$

In terms of guided strategy, several reports have examined differences in clinical outcomes for referring patients according to MPI results to coronary revascularization or medical therapy alone. ${ }^{7-9}$ These results suggest that there may be a threshold whereby patients with moderate to severe ischemia may benefit in terms of reduced mortality and CAD events when a revascularization strategy is applied vs as compared with medical therapy alone. ${ }^{7}$ In addition, important findings outlined that patients undergoing to percutaneous coronary intervention plus medical therapy showed a greater reduction in ischemia with associate lower risk of cardiovascular events as compared to those treated with only medical therapy. ${ }^{10}$ Moreover, in patients with stable disease it has been demonstrated that worsening of myocardial ischemia more than 5\% represent an independent predictor of cardiac events. ${ }^{9}$ All these studies outlined not only the clinical impact of MPI in risk stratify patients with suspected and known CAD but also its important role in related imaging guided therapy. The clinical impact to have a reproducible quantification process helping in the serial follow-up imaging evaluation of CAD patients also emerged. ${ }^{11}$

\section{CZT AND PROGNOSTIC VALUE: WHAT'S NEW?}

Cadmium zinc telluride (CZT) cameras since their introduction showed the possibility to provide a faster image acquisition protocol and lower radiation doses in comparison to traditional sodium-iodine Anger (A) SPECT. The diagnostic value of CZT scanners were described in several studies. ${ }^{12,13}$ Despite both cameras showed a good diagnostic performance in detecting 
angiographic $\mathrm{CAD}$, a slightly higher accuracy by CZTSPECT compared to A-SPECT it emerged. ${ }^{13}$ In some specific categories of patients, such as women at low likelihood of CAD, CZT camera demonstrated a better interpretation of MPI results, due to the better physical performance and image quality. ${ }^{14}$ Prognostic value of CZT cameras have been also recently investigated in some studies. ${ }^{15-17}$ In a population of 1109 consecutive patients with suspected or known CAD Chowdhury et al. found that the cardiac event rate at a follow-up time of 1.7 years was $0.4 \%$ in patients with no significant perfusion abnormalities and $6.8 \%$ in presence of abnormal MPI. ${ }^{15}$ Moreover, impaired resting LV ejection fraction and the presence of significant inducible perfusion defects were independent predictors of poor outcome. ${ }^{15}$ An excellent prognostic value was demonstrated in a consecutive population of 4057 patients with suspected CAD. ${ }^{16}$ In particular, patients with normal perfusion demonstrated a low annual event rate $(0.2 \%)$ increasing with the extent of abnormality of myocardial perfusion that resulted independently associated with an increased risk of events. ${ }^{16}$ Lima et al. using a low-dose protocol, in a high number of patients enrolled, although most asymptomatic and with normal function, found a correlation between increasing mortality and extent of abnormal perfusion. ${ }^{17}$ The prognostic value of the CZT camera has also been studied in stress-first imaging protocols ${ }^{18}$ by low-dose CZT scan in emergency department demonstrating the excellent prognosis of a normal MPI imaging results. ${ }^{18}$ As well, in obese patients, a single-day rest/stress CZT-SPECT with a multi pin-hole camera provided a significant prognostic discrimination with high images quality. ${ }^{19}$

In the current issue of the Journal Miller et al. ${ }^{20}$ evaluated differences in risk prediction between CZT camera and traditional camera, hypothesizing that patients with normal perfusion assessed with a CZT camera system may have a more favorable prognosis due to the higher spatial resolution. The authors analyzed 12,830 consecutive patients, of those 5072 underwent CZT imaging, followed for about 7 years. Increasing stress perfusion abnormalities was associated to an increased risk of all-cause mortality for both CZT and traditional systems. However, patients imaged with a CZT camera compared to those undergoing A-camera imaging were less likely to experience all-cause mortality in patients with normal results and in each category of perfusion abnormality. In addition, the authors performed a propensity match analysis in order to compare possible differences in defect classification between camera system. This was done accordingly to the hypothesis that the higher spatial resolution and sensitivity of CZT camera could influence the difference in prognostication of perfusion abnormality as compared to traditional camera. In the propensity score analysis, authors showed that only patients with normal stress perfusion by CZT camera system were less likely to die during the follow-up compared to patients with normal perfusion by anger system. The strengths of this work were represented by the high number of patients evaluated at long follow-up time although images were not acquired in the same population with both camera system.

Similarly, Lima et al. ${ }^{21}$ compared traditional camera and CZT-SPECT by low-dose protocol in a large patient population performing two totally different protocols and matching population for baseline characteristics but during a follow-up period of 34 months. The CZT camera using a smaller radiation dose and shorter acquisition time showed similar utility to conventional A-camera in prognostication, but also showing a lower prevalence by CZT of hard events in patients with normal scan. ${ }^{21}$ Other studies have evaluated a prognostic comparison between the two SPECT systems, confirming similar value of CZT as compared to traditional system..$^{22,23}$

\section{PROBLEMS SOLVING AND FUTURE DIRECTIONS}

New technology requires a well-defined body of evidence on its benefit in terms of prognosis in order to support and improve the widespread use in routinely clinical application. The estimation of prognosis lead to linkage with patient risk and therapeutic risk reduction effort. Most of the study on the prognostic value of CZT camera is single-center study, involving population with different pretest likelihood of CAD and using different cameras and imaging protocols. Indeed, CZT cameras differ significantly in design and should not been excluded that different CZT cameras may have different prognostic ability. Recently, it has been published a rationale and design of REFINE SPECT registry, ${ }^{24}$ the largest multicenter study on the prognostic validation of solid-state cameras. Aim of the study was to create a multicenter registry collecting clinical, imaging and follow-up data for patients who undergo MPI with the latest generation SPECT scanners. Moreover, registry aids to develop novel machine learning methods for automated diagnosis and prediction of prognostic outcomes. To date, 20.418 solid-state MPI scans with collected prognostic data have been included in the registry. Initial results from the REFINE SPECT have demonstrated that quantitative perfusion analysis, measured as total perfusion deficit (TPD), provides accurate risk stratification, in comparison to visual analysis. ${ }^{25}$ Increasing TPD is associated to increasing risk of cardiac events. Interestingly, patients with normal results at visual reading having a TPD $>0$ but $<1 \%$ 
had a $40 \%$ higher risk of events compared to those with a TPD of 0 . These results demonstrated the relevance of even small defects, which may be not recognized by traditional camera for its lower spatial resolution as compared to new solid-state SPECT cameras. Indeed, higher sensitivity of CZT camera should allow for identification of disease that could have been missed by traditional imaging leading to earlier diagnosis and optimal imaging guided treatment associated to a global reduction of cardiac poor outcomes. Accordingly, Miller et al. ${ }^{20}$ found that patients with normal results at CZT imaging showed lower probability of events in their follow-up as compared to traditional camera showing the higher prognostic value of a negative MPI by CZT imaging. An important point of this study is the length of follow-up considered. The validation of a normal study has important clinical implication using a long-term timing. Further studies are needed to validate prognostic value of CZT camera and ability of risk prediction by using these new dedicated cameras allowing highquality images with a very low radiation dose. Improvements in risk prediction may be relevant, allowing to better detection of patient risk. Moreover, it could be relevant establish the prognostic power of CZT technologies adding the evaluation of myocardial flow reserve, which have demonstrated to provide a high accuracy in identifying the presence of obstructive CAD. ${ }^{26}$ The real power of CZT camera in risk stratify patients also in comparison to traditional camera should be reached adding dynamic flow parameters using SPECT timing reducing low-dose protocol.

\section{References}

1. Nakanishi R, Osawa K, Ceponiene I, Huth G, Cole J, Kim M, et al. The diagnostic performance of SPECT-MPI to predict functional significant coronary artery disease by fractional flow reserve derived from CCTA (FFR(CT)): Sub-analysis from ACCURACY and VCT001 studies. Int J Cardiovasc Imaging 2017;33:2067-72.

2. Shaw LJ, Iskandrian AE. Prognostic value of gated myocardial perfusion SPECT. J Nucl Cardiol 2004;11:171-85.

3. Cremer P, Hachamovitch R, Tamarappoo B. Clinical decision making with myocardial perfusion imaging in patients with known or suspected coronary artery disease. Semin Nucl Med 2014:44:320-9.

4. Panza JA, Holly TA, Asch FM, et al. Inducible myocardial ischemia and outcomes in patients with coronary artery disease and left ventricular dysfunction. J Am Coll Cardiol 2013;61:186070.

5. Shaw LJ, Berman DS, Picard MH, et al. Comparative definitions for moderate-severe ischemia in stress nuclear, echocardiography, and magnetic resonance imaging. J Am Coll Cardiol Cardiovasc Imaging 2014;7:593-604.

6. Bourque JM, Beller GA. Stress myocardial perfusion imaging for assessing prognosis: An update. JACC Cardiovasc Imaging 2011;4:1305-19.
7. Hachamovitch R, Rozanski A, Shaw LJ, et al. Impact of ischaemia and scar on the therapeutic benefit derived from myocardial revascularization vs. medical therapy among patients undergoing stress-rest myocardial perfusion scintigraphy. Eur Heart J 2011;32:1012-24.

8. Petretta M, Acampa W, Daniele S, et al. Long-term survival benefit of coronary revascularization in patients undergoing stress myocardial perfusion imaging. Circ J 2016;80:485-93.

9. Farzaneh-Far A, Phillips HR, Shaw LK, Starr AZ, Fiuzat M, O'Connor CM, et al. Ischemia change in stable coronary artery disease is an independent predictor of death and myocardial infarction. JACC Cardiovasc Imaging 2012;5:715-24.

10. Shaw LJ, Berman DS, Maron DJ, Mancini GB, Hayes SW, Hartigan PM, et al. COURAGE Investigators. Optimal medical therapy with or without percutaneous coronary intervention to reduce ischemic burden: Results from the Clinical Outcomes Utilizing Revascularization and Aggressive Drug Evaluation (COURAGE) trial nuclear substudy. Circulation 2008;117:128391.

11. Iskandrian AE, Hage FG, Shaw LJ, Mahmarian JJ, Berman DS. Serial myocardial perfusion imaging: Defining a significant change and targeting management decisions. JACC Cardiovasc Imaging 2014;7:79-96.

12. Nudi F, Iskandrian AE, Schillaci O, Peruzzi M, Frati G, BiondiZoccai G. Diagnostic accuracy of myocardial perfusion imaging with CZT technology: Systemic review and meta-analysis of comparison with invasive coronary angiography. JACC Cardiovasc Imaging 2017;10:787-94.

13. Cantoni V, Green R, Acampa W, Zampella E, Assante R, Nappi C, et al. Diagnostic performance of myocardial perfusion imaging with conventional and CZT single-photon emission computed tomography in detecting coronary artery disease: A meta-analysis. J Nucl Cardiol 2019. https://doi.org/10.1007/s12350-019-01747-3.

14. Mannarino T, Assante R, Ricciardi C, Zampella E, Nappi C, Gaudieri V, et al. Head-to-head comparison of diagnostic accuracy of stress-only myocardial perfusion imaging with conventional and cadmium-zinc telluride single-photon emission computed tomography in women with suspected coronary artery disease. J Nucl Cardiol 2019. https://doi.org/10.1007/s12350-019-01789-7.

15. Chowdhury FU, Vaidyanathan S, Bould M, Marsh J, Trickett C, Dodds $\mathrm{K}$, et al. Rapid-acquisition myocardial perfusion scintigraphy (MPS) on a novel gamma camera using multipinhole collimation and miniaturized cadmium-zinc-telluride (CZT) detectors: Prognostic value and diagnostic accuracy in a realworld' nuclear cardiology service. Eur Heart J Cardiovasc Imaging 2014; $15: 275-83$.

16. Engbers EM, Timmer JR, Mouden M, Knollema S, Jager PL, Ottervanger JP. Prognostic value of myocardial perfusion imaging with a cadmium-zinc-telluride SPECT camera in patients suspected of having coronary artery disease. J Nucl Med 2017;58:1459-63.

17. Lima RSL, Peclat TR, Souza ACAH, Nakamoto AMK, Neves FM, Souza VF, et al. Prognostic value of a faster, low-radiation myocardial perfusion SPECT protocol in a CZT camera. Int $\mathrm{J}$ Cardiovasc Imaging 2017;33:2049-56.

18. Mouden M, Timmer JR, Ottervanger JP, et al. Impact of a new ultrafast CZT SPECT camera for myocardial perfusion imaging: Fewer equivocal results and lower radiation dose. Eur J Nucl Med Mol Imaging 2012;39:1048-55.

19. De Lorenzo A, Peclat T, Amaral AC, Lima RSL. Prognostic evaluation in obese patients using a dedicated multipinhole cadmium-zinc telluride SPECT camera. Int $\mathrm{J}$ Cardiovasc Imaging 2016;32:355-61. 
20. Miller R., Han D., Rozanski A., Gransar H., John D, Friedman J., et al. CZT Camera Systems May Provide Better Risk Stratification for Low Risk Patients Nucl Cardiol 2020 (in press).

21. Lima R, Peclat T, Soares T, Ferreira C, Souza AC, Camargo G. Comparison of the prognostic value of myocardial perfusion imaging using a CZT-SPECT camera with a conventional anger camera. J Nucl Cardiol 2017;24:245-51.

22. Oldan JD, Shaw LK, Hofmann P, Phelan M, Nelson J, Pagnanelli $\mathrm{R}$, et al. Prognostic value of the cadmium-zinc-telluride camera: A comparison with a conventional (Anger) camera. J Nucl Cardiol 2016;23:1280-7.

23. Yokota S, Mouden M, Ottervanger JP, Engbers E, Knollema S, Timmer JR, et al. Prognostic value of normal stress-only myocardial perfusion imaging: A comparison between conventional and CZT-based SPECT. Eur J Nucl Med Mol Imaging 2016;43:296-301.
24. Slomka PJ, Betancur J, Liang JX, Otaki Y, Hu LH, Sharir T, et al. Rationale and design of the REgistry of Fast Myocardial Perfusion Imaging with Next generation SPECT (REFINE SPECT). J Nucl Cardiol 2018. https://doi.org/10.1007/s12350-018-1326-4.

25. Otaki Y, Betancur J, Sharir T, Hu LH, Gransar H, Liang JX, et al. 5-year prognostic value of quantitative versus visual MPI in subtle perfusion defects: Results from REFINE SPECT. JACC Cardiovasc Imaging 2020;13:774-85.

26. Acampa W, Assante R, Mannarino T, Zampella E, D'Antonio A, Buongiorno $\mathrm{P}$, et al. Low-dose dynamic myocardial perfusion imaging by CZT-SPECT in the identification of obstructive coronary artery disease. Eur J Nucl Med Mol Imaging 2019. h ttps://doi.org/10.1007/s00259-019-04644-6.

Publisher's Note Springer Nature remains neutral with regard to jurisdictional claims in published maps and institutional affiliations. 